\title{
SEPARATION AND VON NEUMANN INTERSECTION THEOREMS
}

\author{
SHIOW-YU CHANG
}

(Communicated by William J. Davis)

\begin{abstract}
We give some separation theorems to extend the intersection theorem of von Neumann, Fan, and others, omitting hypotheses of convexity and local convexity for one of the coordinate spaces.
\end{abstract}

Let $X, Y$ be two nonempty compact convex sets, each in a Euclidean space. Let $E, F$ be closed subsets of $X \times Y$. Von Neumann's intersection theorem [6] asserts that, if for each $x \in X$ and $y \in Y$, both $E(y)=\left\{x^{\prime} \in X \mid\left(x^{\prime}, y\right) \in E\right\}$ and $F(x)=\left\{y^{\prime} \in Y \mid\left(x, y^{\prime}\right) \in F\right\}$ are nonempty and convex, then $E \cap F \neq \varnothing$. For the case where $Y=X$ and $F=\{(x, x) \mid x \in X\}$, this theorem reduces to Kakutani's fixed-point theorem [4]. Ky Fan extended the theorem to locally convex spaces in 1952 [1]. As a further extension, we prove the theorem allowing $X$ or $Y$ to be nonconvex in a nonlocally convex topological vector space.

Our main result is Theorem 3, a generalization of von Neumann's intersection theorem [6]. Its proof relies on some conclusions about separating disjoint graphs, which are stated below as Theorems 1 and 2 . These elementary theorems may find additional uses of interest. Theorem 4 is a generalization of von Neumann's minimax theorem [5].

Let $X, Y$ be topological spaces. A correspondence $U: X \rightarrow Y$ is a function from $X$ to the family of subsets of $Y$. A correspondence $U: X \rightarrow Y$ is said to be with open graph (closed graph) if the set $\operatorname{Gr} U=\{(x, y) \in X \times Y \mid y \in$ $U(x)\}$ is open(closed) in $X \times Y$. We identify $U$ and $\operatorname{Gr} U$. Let $U^{-1}(y)=$ $\{x \in X \mid y \in U(x)\}$. Let $\operatorname{co} S$ denote the convex hull of the set $S$ and Conv $S$ denote the convex closure of the set $S$ where $S$ is a subset of a topological vector space, conventionally Hausdorff. The empty set is both convex and compact.

The following is a separation theorem, which is a stronger form of Hausdorff separation principle.

Theorem 1. Let $X$ be a compact subset of a topological vector space $E_{1}$ and $Y$ a compact subset of a locally convex space $E_{2}$. Let $U$ and $D$ be two correspondences from $X$ into $Y$ of closed graphs such that for each $x \in X$,

Received by the editors February 3, 1989 and, in revised form, June 6, 1989.

1980 Mathematics Subject Classification (1985 Revision). Primary 54H25. 
Conv $U(x) \cap$ Conv $D(x)=\varnothing$ and either Conv $U(x)$ or Conv $D(x)$ is compact. Then there exist two correspondences $G^{\prime}$ and $G^{\prime \prime}$ from $E_{1}$ into $E_{2}$ of open graphs such that

(1) $U \subset G^{\prime}$ and $D \subset G^{\prime \prime}$;

(2) $G^{\prime} \cap G^{\prime \prime}=\varnothing$; and

(3) $G^{\prime}(x)$ and $G^{\prime \prime}(x)$ are convex for all $x \in E_{1}$.

Proof. For each $x \in X$ there exists a neighborhood $W_{x}$ of 0 in $E_{2}$ such that

(a) [Conv $\left.U(x)+W_{x}\right] \cap$ [Conv $\left.D(x)+W_{x}\right]=\varnothing$. We adopt the convention that $\varnothing+W_{x}=\varnothing$. Let $V_{x}^{\prime}$ be a convex open neighborhood of 0 in $E_{2}$ such that

(b) $V_{x}^{\prime}+V_{x}^{\prime} \subset W_{x}$. Since $U$ and $D$ are closed and hence upper semicontinuous, there exists an open, balanced neighborhood $V_{x}$ of 0 in $E_{1}$ such that $U\left(x+V_{x}\right) \subset U(x)+V_{x}^{\prime}$ and $D\left(x+V_{x}\right) \subset D(x)+V_{x}^{\prime}$. Let $V_{x}^{\prime \prime}$ be an open, balanced neighborhood of 0 in $E_{1}$ such that $v_{x}^{\prime \prime}+V_{x}^{\prime \prime}+V_{x}^{\prime \prime} \subset V_{x}$. By compactness there exist finitely many points $x_{1}, \ldots, x_{n} \in X$ such that $X \subset \bigcup_{i=1}^{n}\left(x_{i}+V_{x_{i}}^{\prime \prime}\right)$. Set $V=\bigcap_{i=1}^{n} V_{x_{i}}^{\prime \prime}, V^{\prime}=\bigcap_{i=1}^{n} V_{x_{i}}^{\prime}$.

Since $G_{1}=U+\left(V \times V^{\prime}\right)$ and $G_{2}=D+\left(V \times V^{\prime}\right)$ are open correspondences from $E_{1}$ into $E_{2}$, so are

$$
\begin{aligned}
G^{\prime} & =\left\{(x, y): x \in E_{1} \text { and } y \in \operatorname{co} G_{1}(x)\right\}, \\
G^{\prime \prime} & =\left\{(x, y): x \in E_{1} \text { and } y \in \operatorname{co} G_{2}(x)\right\} .
\end{aligned}
$$

Moreover, $G^{\prime}$ and $G^{\prime \prime}$ clearly have convex values and contain $U$ and $D$, respectively. Now suppose for some $x \in E_{1}$ there exist $\left(x, y^{\prime}\right) \in G^{\prime}$ and $\left(x, y^{\prime \prime}\right) \in G^{\prime \prime}$. Then

$$
y^{\prime} \in \operatorname{co} G_{1}(x)=\operatorname{co}\left(\bigcup_{x^{\prime} \in x+V}\left[U\left(x^{\prime}\right)+V^{\prime}\right]\right)=\operatorname{co}\left(U(x+V)+V^{\prime}\right) .
$$

Thus $U(x+V)$ is nonempty and there exists $x^{\prime} \in(x+V) \cap X$. Fix $j$, $1 \leq j \leq n$, such that $x^{\prime} \in x_{j}+V_{x_{j}}^{\prime \prime}$, and note that $x+V \subset\left(x^{\prime}+V\right)+V \subset$ $x_{j}+V_{x_{j}}^{\prime \prime}+V+V \subset x_{j}+V_{x_{j}}$. It follows that

$$
\begin{aligned}
y^{\prime} \in \operatorname{co}\left(U\left(x_{j}+V_{x_{j}}\right)+V^{\prime}\right) & \subset \operatorname{co}\left(U\left(x_{j}\right)+V_{x_{j}}^{\prime}+V^{\prime}\right) \\
& \subset\left[\operatorname{co} U\left(x_{j}\right)\right]+V_{x_{j}}^{\prime}+V_{x_{j}}^{\prime},
\end{aligned}
$$

since the sum of convex sets is convex. Symmetrically, $y^{\prime \prime} \in\left[\operatorname{co} D\left(x_{j}\right)\right]+V_{x_{j}}^{\prime}+$ $V_{x_{j}}^{\prime}$. Therefore $y^{\prime}$ and $y^{\prime \prime}$ are distinct, belonging to disjoint sets by (b) and (a). Consequently, $G^{\prime}$ and $G^{\prime \prime}$ are disjoint, and the proof is complete.

The following open correspondence separation theorem yields a single-valued continuous selection function and allows one of the original correspondences to have nonconvex values. 
Theorem 2. Let $X$ be a compact subset of a topological vector space $E_{1}$ and $Y$ a compact subset of a locally convex space $E_{2}$. Let the correspondences $U$ and $D$ from $X$ into $Y$ with closed graphs be such that

$$
U \cap D=\varnothing \text { and } U(x) \text { is convex for all } x \in X .
$$

Then there exists a correspondence $G$ from $E_{1}$ into $E_{2}$ of open graph such that

(1) $U \subset G$;

(2) $G \cap D=\varnothing$; and

(3) $G(x)$ is convex for all $x \in X$.

Furthermore, there exist a finite subset $S$ of $Y$ and a (single-valued) continuous function $f: X^{\prime} \rightarrow \operatorname{coS}$ such that $f(x) \in G(x)$ for each $x \in X^{\prime}$, where $X^{\prime}=$ $\{x \in X \mid U(x) \neq \varnothing\}$.

Proof. By Theorem 1, for each $(x, y) \in D$ there exist two open sets $G_{(x, y)}^{\prime}$ and $G_{(x, y)}^{\prime \prime}$ in $X \times Y$ such that

(i) $U \subset G_{(x, y)}^{\prime}$ and $\{(x, y)\} \subset G_{(x, y)}^{\prime \prime}$;

(ii) $G_{(x, y)}^{\prime} \cap G_{(x, y)}^{\prime \prime}=\varnothing$; and

(iii) $G_{(x, y)}^{\prime}\left(x^{\prime}\right)$ is convex for all $x^{\prime} \in X$.

Then $D \subset \bigcup_{(x, y) \in D} G_{(x, y)}^{\prime \prime}$. Since $D$ is compact, there exists a finite subcover $\left\{G_{\left(x_{i}, y_{i}\right)}^{\prime \prime}\right\}_{i=1}^{k}$ of $D$. Let $G=\bigcap_{i=1}^{k} G_{\left(x_{i}, y_{i}\right)}^{\prime}$. Then $G \cap G_{\left(x_{i}, y_{i}\right)}^{\prime \prime}=\varnothing$ for all $i=1, \ldots, k$ and hence $G \cap D=\varnothing$. By the properties of $G_{\left(x_{i}, y_{i}\right)}^{\prime}, U \subset G, G$ is open in $X \times Y$, and $G(x)$ is convex for all $x \in X$.

Since $G$ is open and $U$ is compact, there is a finite set $S \subset Y$ such that $G(x) \cap S \neq \varnothing$ for each $x \in X^{\prime}$. Define $H: X^{\prime} \rightarrow \operatorname{co} S$ by $H(x)=G(x) \cap \operatorname{co} S$. Then $H$ is with open graph and convex nonempty values. By Theorem 3.1 in [7], there is a continuous function $f: X^{\prime} \rightarrow \operatorname{co} S$ such that $f(x) \in H(x)$ for all $x \in X^{\prime}$. This completes the proof.

Theorem 3. Let $X$ be a compact subset of a topological vector space $E_{1}$ and $Y$ a convex compact subset of a locally convex space $E_{2}$. Let $U$ and $D$ be two correspondences with closed graphs from $X$ into $Y$ such that $U(x)$ is nonempty and convex for all $x \in X$ and $D^{-1}(y)$ is nonempty and convex for all $y \in Y$. Then $U \cap D \neq \varnothing$.

Proof. Suppose that $U \cap D=\varnothing$. By Theorem 2 there is a correspondence $G$ from $E_{1}$ into $E_{2}$ with open graph such that

(1) $U \subset G$;

(2) $G \cap D=\varnothing$; and

(3) $G(x)$ is convex for all $x \in X$.

Furthermore, there exist a finite subset $S$ of $Y$ and a (single-valued) continuous function $f: X \rightarrow \cos$ such that $f(x) \in G(x)$ for each $x \in X$. Define $q: \operatorname{co} S \rightarrow X$ by $q(y)=D^{-1}(y)$ for all $y \in \operatorname{co} S$. Then by relaxing $n$-simplex 
to $\operatorname{co} S$ in Lemma 2 of [3], there exists $z \in \cos$ such that $z \in f(q(z))$. This implies that $G \cap D \neq \varnothing$, which is a contradiction, and hence $U \cap D \neq \varnothing$.

Using Theorem 3, one can easily prove:

Theorem 4. Let $X$ be a compact subset of a topological vector space $E_{1}$ and $Y$ a compact convex subset of a locally convex space $E_{2}$. Let $f$ be a real-valued continuous function on $X \times Y$. If, for each $x_{0} \in X, y_{0} \in Y$, the sets:

$$
\left\{x \in X \mid f\left(x, y_{0}\right)=\operatorname{Max}_{\zeta \in X} f\left(\zeta, y_{0}\right)\right\}
$$

and

$$
\left\{y \in Y \mid f\left(x_{0}, y\right)=\operatorname{Min}_{\eta \in Y} f\left(x_{0}, \eta\right)\right\}
$$

are convex, then $\operatorname{Max}_{x \in X} \operatorname{Min}_{y \in Y} f(x, y)=\operatorname{Min}_{y \in Y} \operatorname{Max}_{x \in X} f(x, y)$.

\section{ACKNOWLEDGMENT}

The author would like to thank the referee for suggestions that lead to a better presentation of this paper and a much simpler and shorter Theorem 1 that does not use the Hahn-Banach Theorem.

\section{REFERENCES}

1. K. Fan, Fixed-point and minimax theorems in locally convex topological linear spaces, Proc. Nat. Acad. Sci. U. S. A. 38 (1952), 121-126.

2. I. L. Glicksberg, A further generalization of the Kukutani fixed point theorem, with application to Nash equilibrium points, Proc. Amer. Math. Soc. 3 (1952), 170-174.

3. Chung-Wei Ha, Minimax and fixed point theorem, Math. Ann. 248 (1980), 73-77.

4. S. Kukutani, A generalization of Brouwer's fixed point theorem, Duke Math. J. 7 (1941), 457-459.

5. J. von Neumann, Zur Theorie der Gesellschaftsspiele, Math. Ann. 100 (1928), 295-320.

6. __ Uber ein okonomisches Gleichungssystem und eine Verallgemeinerung des Brouwerschen Fixpunktsatzes, Ergeb. eines Math. 8 (1937), 73-83.

7. N. C. Yannelies and N. D. Prabhakar, Existence of maximal elements and equilibria in linear topological spaces, J. Math. Econom. 12 (1983), 233-245.

Department of Mathematics, Soochow University, Taipei, Taiwan R.O.C. 